\title{
Programming molecular instruments
}

\begin{abstract}
Small conditional RNAs prove their mettle in multiplexed mRNA imaging and show promise as potential cancer therapeutics.

In 2004, Niles Pierce and his colleagues published the proof of principle for a method they termed hybridization chain reaction (HCR). Now, six years later, Pierce's team at the California Institute of Technology presents two exciting and completely unrelated applications.
\end{abstract}

The original version of HCR involved DNA hairpins in a test tube. The hairpins coexist metastably but rapidly polymerize when a hairpin binds to a single-stranded initiator molecule, opening the hairpin. This conformational change exposes the loop portion of the hairpin, which in turn binds to and opens a second hairpin, triggering the formation of a long double-stranded polymer. Though the principle of the technique remains the same today, the new applications no longer use DNA, and the test tube has given way to cells and embryos.

The first application Pierce's team sought to address was the simultaneous imaging of multiple different $m R N A s$ inside intact, fixed zebrafish embryos, an effort spearheaded by graduate student Harry Choi (Choi $e t$ al., 2010). His theory was beautiful: RNA probes bind to the target mRNA and trail single-stranded initiators. The introduction of fluorophore-labeled HCR hairpins leads to 'growth' of fluorescent polymers tethered to the target mRNAs. It turned out, however, that the hairpin design that had worked so well in test tubes was not successful under the stringent hybridization conditions needed to avoid nonspecific binding in vertebrate embryos. Pierce recalls, "for a couple of years, all we would see were black zebrafish with no signal because no polymers were forming."

But rather than deem HCR unfeasible under stringent buffer conditions, Choi and his colleagues performed detailed measurements of the thermodynamic requirements of stable polymer formation. Then they reverseengineered the hairpins to meet these design

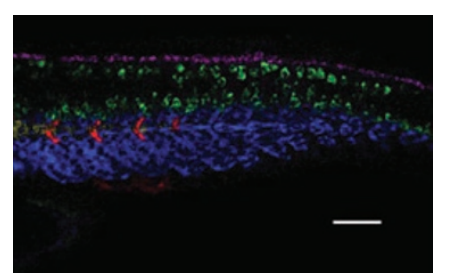

Simultaneous mapping of five target mRNAs within a fixed, whole-mount zebrafish embryo. Scale bar, $50 \mu \mathrm{m}$. Image courtesy of Molecular Instruments.

constraints; instead of using small DNA hairpins, they switched to floppier RNA hairpins. The zebrafish embryos lit up with the expression pattern of the target transcript.

Then they demonstrated the robustness of the technique using five $\mathrm{HCR}$ amplifiers carrying distinct fluorophores to simultaneously map the expression patterns of five target mRNAs in a single intact zebrafish embryo. The hairpins penetrate deep into the embryo and grow polymers only once they encounter the initiator, yielding a high signal-to-background ratio. As the self-assembled polymers are tethered to their target mRNAs, they cannot diffuse away, giving rise to sharp signal localization. Pierce describes this technology as 'ready for prime time' with all parts available from Molecular Instruments.

In parallel, Pierce's team sought to tackle another long-standing challenge-that of treating cancer without the side effects of conventional cancer chemotherapies. "We wanted to see if we could use HCR to engineer chemotherapies that are conditional, activating selectively in cancer cells." says Pierce. In this work, led by graduate student Suvir Venkataraman, RNA hairpins bind to a cancer-specific mRNA translocation, which serves as the initiator and triggers hairpin self-assembly only if the mutation is present in a cell (Venkataraman et al., 2010). Polymer assembly produces long double-stranded RNA molecules that the cancer cell mistakes for viral RNA, triggering an innate immune response leading to cell death.

At the beginning of this project, it was not clear whether HCR would work inside living cells. But after designing RNA hairpins suitable for use in physiological conditions, the researchers proved the principle of this idea. They designed three HCR hairpin sequences suitable for detecting three different translocation mutations, each specific to a different human cancer cell line.

Having demonstrated the principle with translocation mutations, the team is now testing whether HCR hairpins can be designed to selectively kill cancer cells based on recognition of single point mutations.

From killing cells in a dish, they have now moved to mouse models, which will present additional challenges. Effective delivery of the molecules is one challenge, which the researchers hope to meet by taking advantage of technologies developed for therapeutic RNA interference studies. Pierce acknowledges that pursuing conditional chemotherapies based on HCR is a high-risk strategy but says, "looking at the abysmal statistics for progress in treating cancer using conventional approaches, we can't afford not to take risks."

Even if this application should never reach the level of human therapeutics, Pierce thinks that the concept of 'molecular instruments' based on small conditional RNAs will be successful. What he wants to see is a new way to design biology experiments. Pierce's vision is that "biologists should be able to order molecular instruments that are programmed to measure or regulate the cell-state property that they are interested in studying."

Nicole Rusk

\section{RESEARCH PAPERS}

Choi, H.M.T. et al. Programmable in situ amplification for multiplexed imaging of mRNA expression. Nat. Biotechnol. 28, 1208-1212 (2010).

Venkataraman, S. et al. Selective cell death mediated by small conditional RNAs. Proc. Natl. Acad. Sci. USA 107, 16777-16782 (2010). 